\title{
Patterned Optical Trapping with Two-Dimensional Photonic Crystals
}

\author{
Peifeng Jing, Jingda Wu, and Lih Y. Lin* \\ Department of Electrical Engineering, University of Washington, Seattle, Washington 98195, United States
}

ABSTRACT: We demonstrate an approach to achieve patterned optical trapping with two-dimensional photonic crystals (2D PC). A Gaussian beam infrared laser is guided and loosely focused by a simple optical system onto the surface of the 2D PC, which generates an enhanced diffraction field for optical trapping of microbeads with high efficiency. The diffraction patterns are determined by the structures of the $2 \mathrm{D}$ PC, and the experimental results match well with the modeling results using the finite-difference time-domain method. Polarization control is demonstrated as one way to achieve reconfigurability. Using this approach, we demonstrate high design flexibility for patterned optical trapping as determined by the 2D PC structure with low laser intensity.

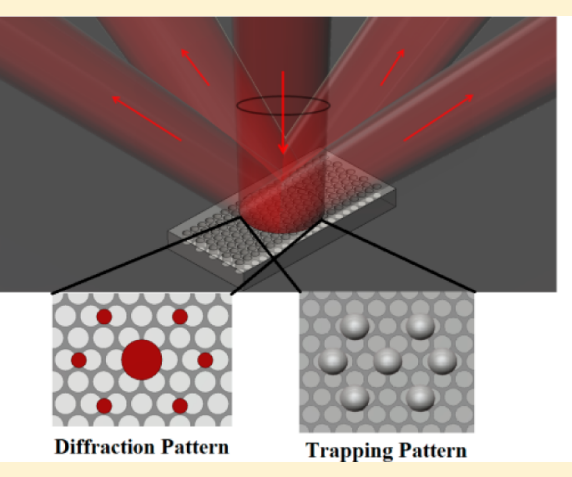

Patterned optical trapping with low intensity is determined by the structure of two-dimensional photonic crystal platform and the diffraction patterns.

KEYWORDS: optical tweezers, $2 D$ photonic crystal, patterned optical trapping

$\mathrm{T}$ he photonic crystal (PC $)^{1}$ has become an important structure for manipulating photons and has demonstrated great success and potential for various applications. Much effort has been made on PC geometry design and fabrication to optimize the photonic band structure for various utilizations, such as light-trapping cavities and resonators, ${ }^{2-4}$ optical fibers, ${ }^{5}$ and waveguides. ${ }^{6}$ Most of these applications utilize the photonic band-gap property of PCs, and light propagates along the direction of the periodic structure. More recently, light emission normal to the surface of the PC structure has been explored. Theoretical study and numerical simulations have shown that the emission pattern from the PC surface can be designed, ${ }^{7}$ and two-dimensional optical feedback has been utilized to engineer PC structures for compact semiconductor lasers that produce a range of beam patterns. ${ }^{8,9}$

Optical tweezers are another significant development in photonics. Since their inception, optical tweezers have proven to be a powerful tool for biological applications and nanotechnology due to their capability of trapping and positioning micro- and nanoparticles with high precision, flexibility, and noninvasiveness. ${ }^{10,11}$ Recently, the capability of manipulating photons and the optical field through PCs has found its application in optical tweezers. The concentrated optical near-field along a PC waveguide surface has been shown to be able to trap small particles efficiently. ${ }^{12,13}$ Utilizing the enhanced diffraction pattern from the surface of a 1D PC nanostructure, we have also demonstrated trapping and aligning particles with a wide range of size from $10 \mu \mathrm{m}$ down to $190 \mathrm{~nm}$ with lower optical intensity than conventional optical tweezers. ${ }^{14}$ Trapping of ovarian cancer cell nuclei was achieved with $16 \mu \mathrm{W} / \mu \mathrm{m}^{2}$ intensity, and Listeria cells were trapped and aligned with an optical intensity of $40 \mu \mathrm{W} / \mu \mathrm{m}^{2}$. Trapping particles in various patterns is desirable for the functionality and performance of optical tweezers. The typical approach, the holographic optical tweezers, is using a spatial light modulator to generate the optical patterns. ${ }^{15,16}$ While this method can achieve versatile optical trap patterns, exquisite optical setups and high optical intensities are often required. The latter particularly limits its application in biological studies.

In this work, we propose and demonstrate an approach of optical manipulation utilizing the interaction of a laser beam with a $2 \mathrm{D}$ PC. A simple optical setup is used to guide a loosely focused laser beam onto the surface of a 2D PC through a microscope, which then generates a patterned optical diffraction field for enhanced trapping. We observe diffraction patterns of the $1064 \mathrm{~nm} \mathrm{Nd}: \mathrm{YVO}_{4}$ laser near the surface of the 2D PC, which match well with the simulation results using a finite-difference time-domain (FDTD) method. In addition, we show that microbeads can be trapped in the same pattern with low optical intensity due to the enhanced optical field near the PC surface. The trapping pattern is determined by the structure of the 2D PC regardless of the size of the laser beam. The utilization of the 2D PC may provide an alternative route for patterned optical trapping with low laser intensity and can complement and increase the capability of holographic optical tweezers by engineering the trap structures generated from each single optical beam.

\section{RESULTS AND DISCUSSION}

Figure 1 shows the schematic drawing of the proposed approach. A loosely focused laser beam is incident upon the surface of the PC, producing a $2 \mathrm{D}$ diffraction pattern for optical trapping. The details of the experimental setup and the incident laser intensities are described in the Methods section. Figure 2 shows an optical microscope image of the 2D PC structure.

Received: September 6, 2013

Published: April 14, 2014 


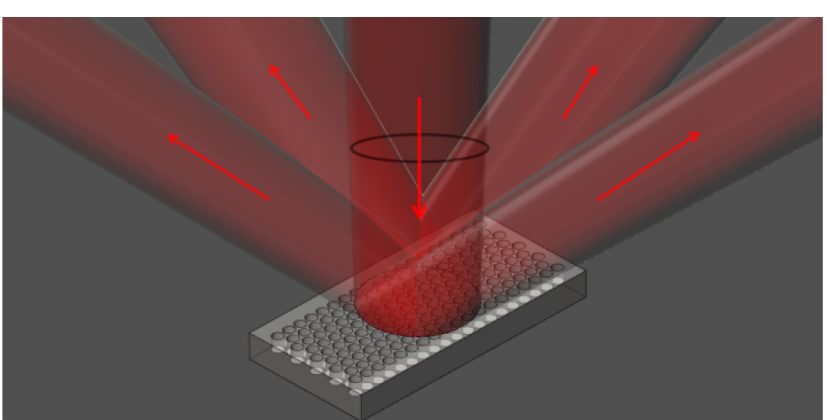

Figure 1. Schematic drawing of the proposed patterned optical trapping on a $2 \mathrm{D}$ PC platform.

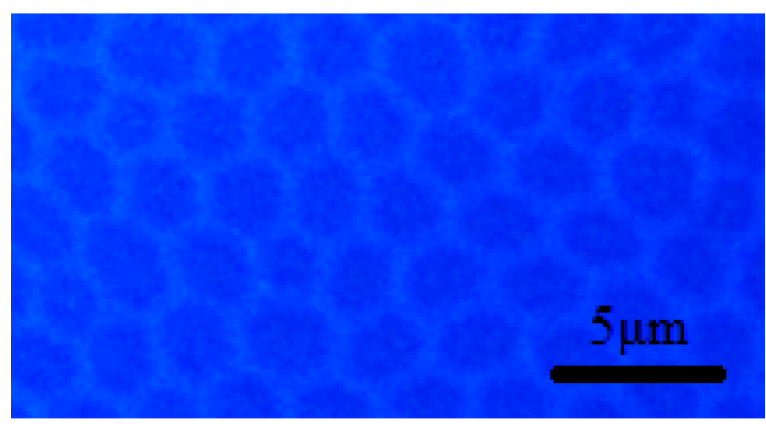

Figure 2. Top-view optical image of the 2D photonic crystal used for patterned optical trapping.

Although it does not have complete periodically ordered structure as typical PCs, literature has shown that such hyperuniform PC structure with short-range geometric order can still achieve complete photonic band gaps, ${ }^{17}$ and it can alleviate the requirement of e-beam lithography significantly.

We use the MEEP FDTD solver for modeling the diffraction patterns from the 2D PC and compare it with experimental results. The comparison between simulation and experimental results can support the conclusion that the trapping pattern is determined by the structure of the 2D PC. Figure 3 shows one example. In the modeling, the size of the single cell in the PC is set as what is used in the experiment $(2.9 \mu \mathrm{m})$, and the Gaussian beam laser profile covers three holes. A typical regular

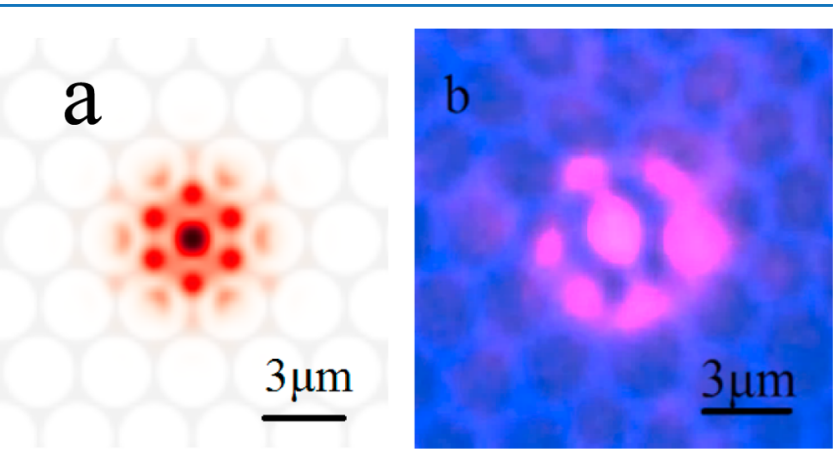

Figure 3. (a) FDTD simulation using MEEP. The dimension of a single cell in the PC is $2.9 \mu \mathrm{m}$, and each hole is cylindrical in shape. The background shows the 2D PC. The incident light is set as a Gaussian laser beam with $1064 \mathrm{~nm}$ wavelength, and the beam profile is assumed to be similar to what is observed in this experiment, covering three holes. (b) Light intensity distribution above the surface of the $2 \mathrm{D}$ PC under a $50 \times$ objectives lens, showing $2 \mathrm{D}$ diffraction by the periodic structure of the PC. hexagonal PC structure is assumed for the modeling. The images of diffracted light intensity distributions and trapping results are recorded by a CCD camera connected to the microscope. Figure $3 \mathrm{~b}$ shows the experimental result of the diffracted beam profile under the $50 \times$ objective lens. Although the PC platform is a hyperuniform PC structure with shortrange order as shown in the background of Figure $3 b$, the simulation result (Figure 3a) and experimental result (Figure 3b) match each other reasonably well.

To demonstrate the determinant role of the PC in creating trapping patterns and specifically the flexibility of pattern control, we generated different trapping patterns with the same photonic crystal. The diffraction and trapping patterns in Figure 4 are produced by focusing the laser spot in the center of a hole and at the intersection of three holes. In Figure $4 a$ and $b$, the laser spot is set in the center of a hole, which generates a diffraction pattern consisting of a more intense center and six weaker outer spots. The center pattern traps three beads and the outer spots each trap one bead except the spot on the left, which traps two beads simultaneously. The locations of the outer beads are not exactly the same as the diffraction pattern, which may be attributed to the utilization of the hyperuniform PC that does not have exactly the same structure as the regular periodic hexagonal structure used in the simulation. The divergence of the diffracted beams also affects the location of the trapping beads. The laser spot is then moved to the intersection of three holes, which produces a hexagon diffraction pattern with a center maximum and three prominent outer spots. Figure $4 \mathrm{c}$ shows the FDTD modeling result, and Figure $4 \mathrm{~d}$ shows the patterned trapping of the microbeads, which agree with the modeling result well. More complex trap arrays and reconfigurability can be achieved by incorporating a spatial light modulator or polarization control in the optical system. Here we demonstrate one way to dynamically reconfigure the trap pattern using polarization control. The 1 $\mu \mathrm{m}$ polystyrene beads are first trapped by a linearly polarized laser beam above the surface of the 2D PC, as shown in Figure 4e. When the polarization is rotated 40 degrees, the trap pattern is rotated accordingly (Figure $4 \mathrm{f}$ ). The scale of the pattern is enlarged slightly during the process, which may be caused by the different periodicity of holes in different directions and the semirandom distribution of holes.

To characterize the trapping efficiency, the stiffness and minimum trapping intensity are measured using polystyrene beads of sizes ranging from $100 \mathrm{~nm}$ to $1 \mu \mathrm{m}$ as the trapped particles. Histograms of the displacement of $1 \mu \mathrm{m}$ beads trapped at the center of the diffraction pattern are shown in Figure $5 \mathrm{a}$ and $\mathrm{b}$. The results are a nearly Gaussian profile, allowing calculation of the stiffness by the equipartition method. ${ }^{12}$ The minimum incident optical intensity to maintain the static trapping is shown in Figure 5c. Two sets of data are shown here. The upper one (represented by the circular data points) shows the measured minimum intensity for trapping the beads in the whole diffraction pattern. The intensity is then reduced, and the beads trapped at the outer part of the pattern start to escape due to Brownian motion. As the intensity is further reduced, the beads trapped at the center of the pattern begin to escape too. The lower curve (represented by the square data points) shows the minimum intensity to maintain a stable center trap. The trapping intensity ranges from $<1 \mu \mathrm{W} /$ $\mu \mathrm{m}^{2}$ for $1 \mu \mathrm{m}$ beads to $\sim 16 \mu \mathrm{W} / \mu \mathrm{m}^{2}$ for $100 \mathrm{~nm}$ beads for the lower curve and $\sim 6 \mu \mathrm{W} / \mu \mathrm{m}^{2}$ for $1 \mu \mathrm{m}$ beads to $\sim 30 \mu \mathrm{W} / \mu \mathrm{m}^{2}$ for $450 \mathrm{~nm}$ beads for the upper curve. These are significantly 

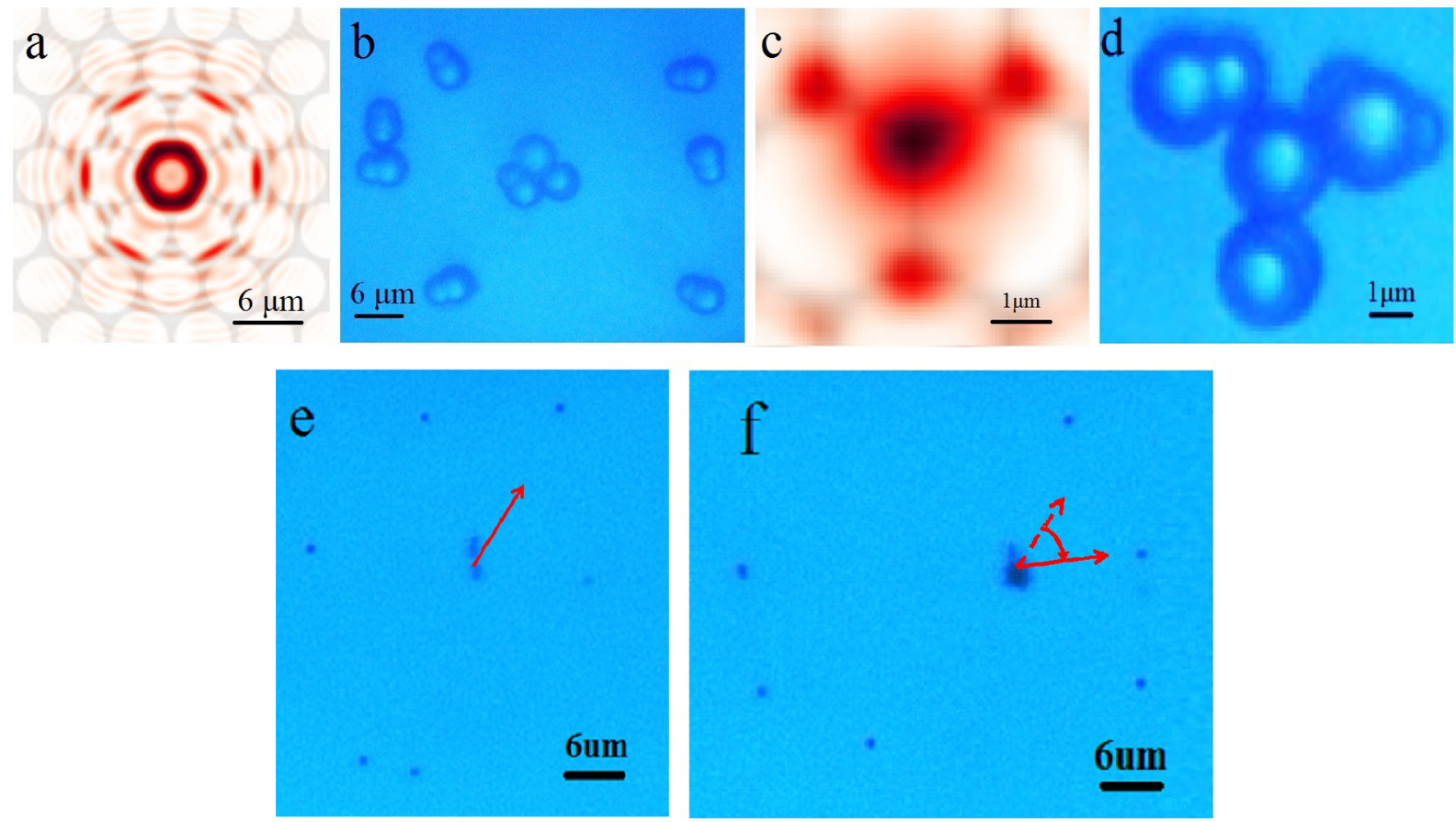

Figure 4. (a) FDTD modeling result with the focused laser spot in the center of a hole, generating a hexagon diffraction pattern. The unit cell size of the 2D PC is $5.8 \mu \mathrm{m}$, which is what is used in this experiment. (b) Patterned optical trapping experimental results of microbeads corresponding to the conditions in part a, except a hyperuniform PC with short-range order is used instead of the regular hexagonal structure assumed in the simulation. (c) FDTD modeling result with the focused laser spot at the intersection of three holes, generating a hexagonal diffraction pattern. (d) Optical trapping experimental results corresponding to the conditions in part c. (e) Trap pattern of $1 \mu \mathrm{m}$ beads generated using a laser beam polarized linearly along the direction indicated by the red arrow. (f) Trap pattern of $1 \mu \mathrm{m}$ beads after the laser polarization is rotated 40 degrees.
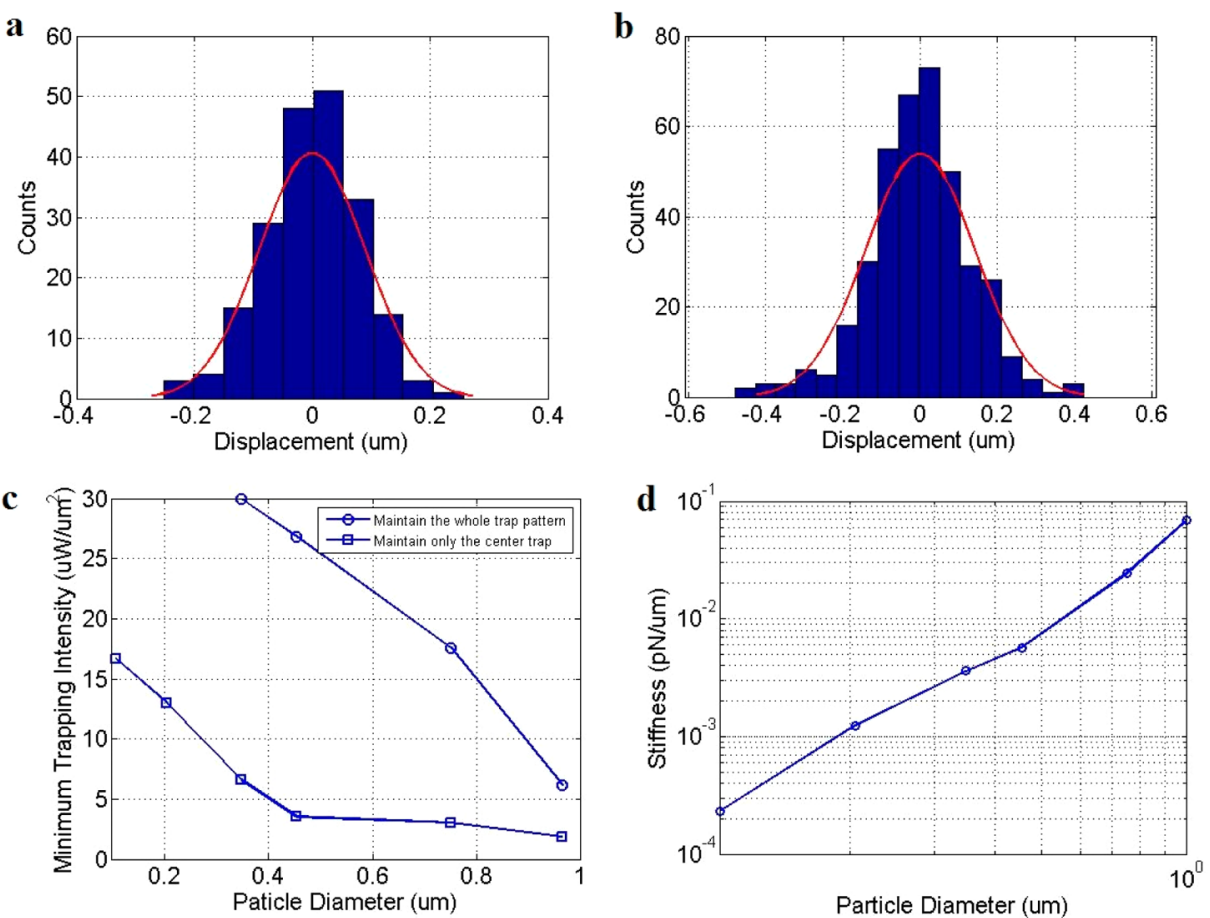

Figure 5. Histogram of displacement of trapped $1 \mu \mathrm{m}$ polystyrene beads (a) in the X direction and (b) in the Y direction. (c and d) Minimum trapping intensity and trap stiffness in the $\mathrm{X}$ direction measured with an input intensity of $3.5 \mu \mathrm{W} / \mu \mathrm{m}^{2}$ for polystyrene beads of various sizes under a $20 \times$ objective lens.

lower than optical intensities used for trapping micrometer-size particles in typical optical tweezers $\left(\sim 1 \mathrm{~mW} / \mu \mathrm{m}^{2}\right) .{ }^{18}$ It is also lower than the results reported in our previous work (ref 14), which used a $633 \mathrm{~nm}$ laser and a 1D PC structure.
The trap stiffness can be obtained by characterizing histograms of displacements under a specific incident optical intensity. For this measurement, it is kept at $3.5 \mu \mathrm{W} / \mu \mathrm{m}^{2}$ under a $20 \times$ objective lens. The results are shown in Figure $5 \mathrm{~d}$. We 

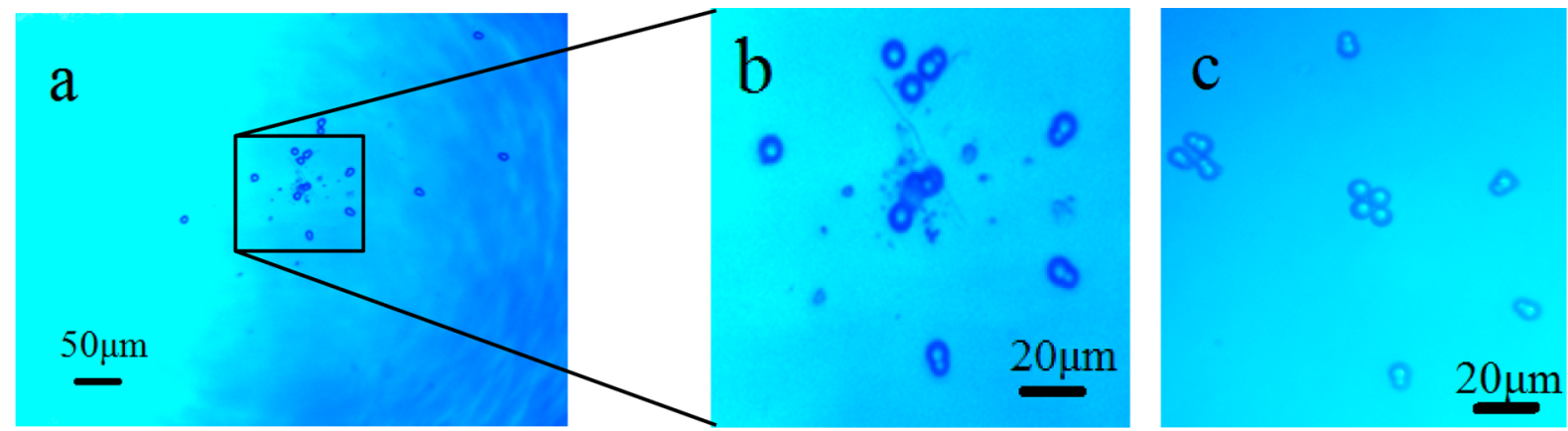

Figure 6. (a) Patterned trapping of microbeads under $20 \times$ objective lenses and (b) enlargement of the center optical trap area. (c) The same trapping experiment but under a 50× objective and therefore smaller focused laser spot size. The result shows a similar trapped bead pattern to that shown in part $b$.

then increase the power to $\sim 17 \mu \mathrm{W} / \mu \mathrm{m}^{2}$ to have a more stable trapping. For $1 \mu \mathrm{m}$ polystyrene beads, the trap stiffness is increased to $0.36 \mathrm{pN} / \mu \mathrm{m}$ in the $\mathrm{X}$ direction and $0.179 \mathrm{pN} / \mu \mathrm{m}$ in the $\mathrm{Y}$ direction. When the same input laser power is focused by a $50 \times$ objective lens, the trap stiffness is $0.387 \mathrm{pN} / \mu \mathrm{m}$ in the $\mathrm{X}$ direction and $0.281 \mathrm{pN} / \mu \mathrm{m}$ in the $\mathrm{Y}$ direction. This result shows that a loosely focused laser beam can achieve almost the same stiffness as a tightly focused beam. Figure $5 \mathrm{~d}$ also shows a linear relation between particle size (in log scale) and the stiffness calculated by the equipartion method. Currently there is a glass spacer of $0.18 \mathrm{~mm}$ thickness between the particles and the PC substrate. The stiffness is expected to be further improved by trapping particles closer to the surface of the $2 \mathrm{D}$ PC.

The scale of the trapping pattern is determined by the structure of the 2D PC regardless of the size of loosely focused beam spots. To investigate the effect of the beam spot size on the patterned optical trapping result, we performed the trapping experiments on the same 2D PC platform with the laser beam focused by the $20 \times$ and $50 \times$ objective lenses. The results are shown in Figure $6 \mathrm{a}$ and c, respectively. Figure $6 \mathrm{~b}$ shows the enlargement of Figure 6 a to the same scale as Figure $6 c$ for comparison. The trapped bead patterns appear to be similar in both cases with the same scale even though the incident laser beam sizes are different. The size of the unit cell is $2.9 \mu \mathrm{m}$ for the $2 \mathrm{D}$ PC structure used in these experiments, which is half of the 2D PC unit cell size used in the experiments generating the results shown in Figure 4. This results in a higher divergence of diffracted beams and therefore wider separations between the trapped beads. Also, compared to Figure $4 b$, one trapping point is missing from the lower-left corner in both Figure $6 \mathrm{~b}$ and $c$. This may be caused by the semirandom distribution of the holes and that a different platform with a different unit cell size and somewhat different PC structure is used. Overall the experimental results support the observation that the optical trap pattern is determined by the structure of the $2 \mathrm{D} \mathrm{PC}$ and how it interacts with the incident laser beam; various patterns with different scales and shapes can be achieved by designing these parameters.

In conclusion, we have shown with both FDTD modeling and experimental demonstration that employing a $2 \mathrm{D} \mathrm{PC}$ as the platform in an optical tweezers system, patterned optical trapping with enhanced efficiency and low light intensities can be achieved. In addition, we show that various trap patterns can be generated with the same photonic crystal by controlling the position of the laser spot. The trapped patterns are determined by the structure of the photonic crystal and independent of the laser spot size. The results show that the proposed approach may provide an effective alternative for patterned optical trapping, complementing the capability of holographic optical tweezers, as this method can be used in a single- or multibeam optical tweezers system. In addition, polarization dependence is shown as a way to implement reconfigurability for this approach. This functionality may have potential applications in biology studies, micro- and nanofabrications, and other areas where patterned manipulation of multiple particles with low optical intensities are desired.

\section{METHODS}

Experimental Setup. Infrared light at a wavelength of 1064 $\mathrm{nm}$ produced by a $\mathrm{Nd}: \mathrm{YVO}_{4}$ laser is used as the light source. It is directed into a Zeiss Axio Imager fluorescence microscope and irradiates normal to the surface of the PC through a beam splitter and an objective lens. The laser beam spot diameter size is about 53 and $25 \mu \mathrm{m}$ when focused by a $20 \times$ objective lens $(\mathrm{N} . \mathrm{A} .=0.22)$ and $50 \times$ objective lens (N.A. $=0.55)$, respectively. For the experiments shown in Figures 4 and 6, the laser power on the PC surface is adjustable and is operated at $8.4 \mathrm{~mW}$ under a $20 \times$ objective lens and $4.3 \mathrm{~mW}$ under a $50 \times$ objective lens in the experiment, which results in an optical intensity of 3.7 and $9 \mu \mathrm{W} / \mu \mathrm{m}^{2}$, respectively. The particles used in the trapping experiments are $1 \mu \mathrm{m}$ diameter polystyrene beads and oblong-shaped polystyrene beads with a long diameter $\sim 6.8 \mu \mathrm{m}$.

Theoretical Study. The effect of trapping microbeads on the 2D PC platform is first determined by intensity distribution of light after diffraction, which can be achieved with FDTD solvers. After obtaining the intensity distribution, the optical forces including gradient force $\vec{F}_{\text {grad }}$ scattering force $\vec{F}_{\text {scat }}$ and absorption force $\vec{F}_{\text {abs }}$ can be calculated for optical trapping. In these three forces, the scattering and absorption forces push particles away from the trapping position and are observed to have little contribution in the experiment compared to the gradient force. The gradient force and the potential energy for optical trapping can be approximately obtained from the laser intensity distribution by ${ }^{19}$

$$
U_{\text {grad }}(x, y, z)=\frac{n_{2}^{2}-n_{1}^{2}}{16 \pi} \int|\vec{E}(x, y, z)|^{2} \mathrm{~d} \nu
$$

The gradient force in the direction of the inhomogeneous field gradient will pull the particles to the laser focus and can be calculated as follows:

$$
\vec{F}_{\text {grad }}(x, y, z)=\nabla U_{\text {grad }}(x, y, z)
$$


The periodic surface structure of a 2D PC makes it behave as a $2 \mathrm{D}$ reflective grating and produce a diffraction trap pattern. But the analysis should not treat the $2 \mathrm{D} P C$ as simple $2 \mathrm{D}$ reflective gratings because the period of holes is comparable to the laser wavelength. Therefore, coupling between optical waves in adjacent holes cannot be ignored, and the coupledwave theory should be recruited for the analysis of the optical field produced by the interaction of light with the $2 \mathrm{D}$ PC. $7,9,20$ Specifically, in a triangular-lattice PC, the slowly varying intensity profile envelope modulates the fast-varying Bloch waves in the PC lattice and can be determined by 9,21

$$
I(x, y)=\sum_{k=1}^{3}\left(\left|R_{k}(x, y)\right|^{2}+\left|S_{k}(x, y)\right|^{2}\right)
$$

The $R_{k}$ and $S_{k}$ are the complex amplitudes of the waves propagating at the second-order $\Gamma$ point as shown in Figure 7.9

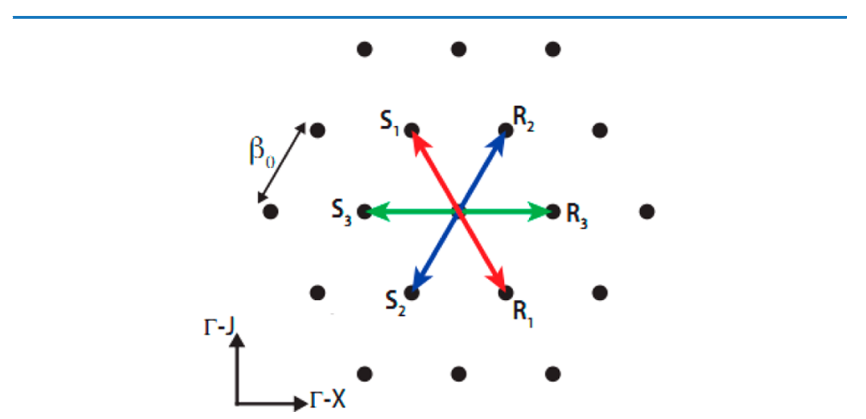

Figure 7. Reciprocal lattice space of a triangular-lattice PC. The colored arrows indicate the six basic waves, $R_{1}, S_{1}, R_{2}, S_{2}, R_{3}$, and $S_{3}$, and the second-order $\Gamma$ point, whose wavenumber is equal to $\beta_{0}=4 \pi /$ $\sqrt{ } 3 a{ }^{9}$

Since this analytical approach is complex, FDTD is a powerful method to achieve the computational diffraction result. We use open-source software MEEP to achieve FDTD numerical results of the intensity profile of the laser beam after reflecting off the surface of the 2D PC.

\section{AUTHOR INFORMATION}

\section{Corresponding Author}

*E-mail: lylin@uw.edu.

\section{Notes}

The authors declare no competing financial interest.

\section{ACKNOWLEDGMENTS}

This work is supported in part by DOD grant OC093221 through Fred Hutchinson Cancer Research Center. We thank Dr. Rick Bjorko of UW Nanotech User Facility for fabricating the 2D PC structures.

\section{REFERENCES}

(1) Yablonovitch, E.; Gmitter, T. J. Photonic band structure: the facecentered-cubic case. Phys. Rev. Lett. 1989, 63, 1950-1953.

(2) Akahane, Y.; Asano, T.; Song, B. S.; Noda, S. High-Q photonic nanocavity in a two-dimensional photonic crystal. Nature 2003, 425, 944-947.

(3) Majumdar, A.; Kim, J.; Vuckovic, J.; Wang, F. Electrical control of silicon photonic crystal cavity by graphene. Nano Lett. 2013, 13, 515518.

(4) Foresi, J. S.; Villeneuve, P. R.; Ferrera, J.; Thoen, E. R.; Steinmeyer, G.; Fan, S.; Joannopoulos, J. D.; Kimerling, L. C.; Smith,
H. I.; Ippen, E. P. Photonic-bandgap microcavities in optical waveguides. Nature 1997, 390, 143-145.

(5) Russell, P. Photonic crystal fibers. Science 2003, 299, 358-362.

(6) Mekis, A.; Chen, J. C.; Kurland, I.; Fan, S.; Villeneuve, P. R.; Joannopoulos, J. D. High transmission through sharp bends in photonic crystal waveguides. Phys. Rev. Lett. 1996, 77, 3787-3790.

(7) Sakai, K.; Miyai, E.; Noda, S. Coupled-wave theory for squarelattice photonic crystal lasers with TE polarization. IEEE J. Quantum Electron. 2010, 46, 788-795.

(8) Miyai, E.; Sakai, K.; Okano, T.; Kunishi, W.; Ohnishi, D.; Noda, S. Photonics: lasers producing tailored beams. Nature 2006, 441, 946.

(9) Liang, Y.; Peng, C.; Ishizaki, K.; Iwahashi, S.; Sakai, K.; Tanaka, Y.; Kitamura, K.; Noda, S. Three-dimensional coupled-wave analysis for triangular-lattice photonic-crystal surface-emitting lasers with transverse electric polarization. Opt. Express 2013, 21, 565-580.

(10) Ashkin, A.; Dziedzic, J. M.; Yamane, T. Optical trapping and manipulation of single cells using infrared laser beams. Nature 1987, 330, 769-771.

(11) Ashkin, A.; Dziedzic, J. M. Optical trapping and manipulation of viruses and bacteria. Science 1987, 235, 1517-1520.

(12) Mandal, S.; Serey, X.; Erickson, D. Nanomanipulation using silicon photonic crystal resonators. Nano Lett. 2010, 10, 99-104.

(13) Chen, Y. F.; Serey, X.; Sarkar, R.; Chen, P.; Erickson, D. Controlled Photonic Manipulation of Proteins and Other Nanomaterials. Nano Lett. 2012, 12, 1633-1637.

(14) Wilson, B. K.; Mentele, T.; Bachar, S.; Knouf, E.; Bendoraite, A.; Tewari, M.; Pun, S. H.; Lin, L. Y. Nanostructure-enhanced laser tweezers for efficient trapping and alignment of particles. Opt. Express 2010, 18, 16005-16013.

(15) Prentice, P. A.; MacDonald, M. P.; Frank, T. G.; Cuschieri, A.; Spalding, G. C.; Sibbett, W.; Campbell, P. A.; Dholakia, K. Manipulation and filtration of low index particles with holographic Laguerre-Gaussian optical trap arrays. Opt. Express 2004, 12, 593-600.

(16) Grier, D. G. A revolution in optical manipulation. Nature 2003, 424, 810-816.

(17) Florescu, M.; Torquato, S.; Steinhardt, P. J. Designer disordered materials with large, complete photonic band gaps. Proc. Natl. Acad. Sci. U.S.A. 2009, 106, 20658-20663.

(18) Wu, M. C. Optoelectronic tweeers. Nat. Photonics 2011, 5, 322324.

(19) Volpe, G.; Quidant, R.; Badenes, G.; Petrov, D. Surface plasmon radiation forces. Phys. Rev. Lett. 2006, 96, 238101.

(20) Liang, Y.; Peng, C.; Sakai, K.; Iwahashi, S.; Noda, S. Threedimensional coupled-wave analysis for square-lattice photonic crystal surface emitting lasers with transverse-electric polarization: finite-size effect. Opt. Express 2012, 20, 15945-15961.

(21) Wang, Y.; Qu, H.; Zhou, W.; Qi, A.; Zhang, J.; Liu, L. Lateral cavity photonic crystal surface emitting laser based on commercial epitaxial wafer. Opt. Express 2013, 21, 8844-8855. 